\title{
La mediación pedagógica en el sistema familiar frente a los contenidos en televisión, internet y redes sociales online*
}

\section{Pedagogical Mediation in the Family System Regarding Contents on Television, the Internet and Online Social Networks}

\author{
Marta Silva Pertuz* \\ ORCID: 0000-0002-3779-8429 \\ Universidad Metropolitana, Barranquilla, \\ Colombia \\ Adriana Silva Silva \\ ORCID: 0000-0002-9584-7284 \\ Universidad Sergio Arboleda, Santa Marta, \\ Colombia \\ Centro de Formación Clínica Psicosocial \\ Filigrana Caribe S.A.S., Barranquilla, \\ Colombia \\ Andrés F. García Jiménez \\ ORCID: 0000-0002-2269-2162 \\ Universidad Metropolitana \\ Corporación Universitaria Reformada, \\ Barranquilla, Colombia
}

Recibido: 22 enero de 2019

Revisado: 7 de junio de 2019 Aceptado: 4 de septiembre 2019

\section{Resumen}

Este artículo es producto de una investigación de mixta donde predominó el énfasis en la descripción de los resultados, con características cuantitativas de la información obtenida a través del cuestionario aplicado. El diseño descriptivo permitió observar y reflexionar sobre las mediaciones pedagógicas que se dan en los contextos familiares frente a la recepción y consumo de productos y servicios ofertados por la televisión, internet y las redes sociales on line. Los participantes fueron estudiantes universitarios (16 y 24 años de edad) a los que se les aplicó un cuestionario y un diario de observación. Se realizó una triangulación de los resultados de la observación participante y de los cuestionarios aplicados. Se encontró que existía un tipo de mediación familiar frente a los contenidos televisivos. Sin embargo, no se encontró un indicador que aclare si las familias están mediando los contenidos que circulan en internet. Esto permite una oportunidad para plantear estrategias de comunicación familiar, que posibiliten una injerencia de los padres / cuidadores o acompañantes sobre estos contenidos, teniendo en cuenta el ciclo vital individual y familiar.

Palabras clave: medios de comunicación social; Mediación familiar; Mediación educativa; Mediación social.

Artículo de Investigación. Citar como: Pertuz, M. S., Silva, S. A., \& García, J. A. F. (2020). La mediación pedagógica en el sistema familiar frente a los contenidos en televisión, internet y redes sociales online. Diversitas: Perspectivas en Psicología, 16(1), 157-168. Dol: https://doi. org/10.15332/22563067.5548

** Autor de correspondencia: Adriana Sofia Silva Silva. Dirección de correspondencia: Calle 33 E \# 2C1-35, Universal. Correo electrónico: adriana. silvas@correo.usa.edu.co, filigranacaribe@gmail.com 


\section{Abstract}

This article was based on a mixed methods research where the predominant emphasis was placed on the description of the results, with quantitative characteristics obtained through the applied questionnaire. The descriptive design made it possible to observe and reflect on the pedagogical mediations that occur in family contexts with regard to the reception and consumption of products and services offered by television, the internet, and online social networks. The participants were university students ( 16 and 24-year-old) who responded the questionnaire and kept an observational diary. A triangulation of the results of both the participants' observations and the questionnaires was used. We found that there is a type of family mediation with regard to television contents. However, no indicator was found to clarify if the families are mediating the contents and information circulating on the internet. This opens up an opportunity to propose family communication strategies that allow for a more direct influence of the parents/caregivers or companions on these contents, taking into account the individual and family life cycles.

Keywords: social media; family and school settings; training scenarios; family mediation; educational mediation; social mediation.

\section{Introducción}

La Internet se convirtió en la década de los noventa en un poderoso canal multimedia de comunicación interpersonal y social, sincrónico (chat, video chat, mensajería instantánea, entre otros) y asincrónico (correo electrónico o foros). Se constituyó en una inmensa y dinámica fuente de información sobre cualquier tema o actividad y un medio de publicación global de noticias, documentos y creaciones personales (páginas web, blogs, wikis, entornos de publicación, $T V$ on-line) al alcance de todos. Se multiplican los canales de información y comunicación, por lo que se hizo necesario iniciar a pensar en las formas posibles para catalizar los aprendizajes, usos racionales y necesarios, que disminuyeran los abusos y dependencias o adicciones tecnológicas para algunos.

Al igual que sucedía y aun ocurre con la televisión, el lugar y uso que ocupa el computador en el hogar sufre variaciones en función del género, la edad, las condiciones socioeconómicas, el nivel educativo, el estilo para ejercer la comunicación, la autoridad y la puesta de límites, entre otros aspectos, factores y categorías. Ha sido evidente que la familia desempeña un papel fundamental y predominante en el establecimiento de pautas de interacción y socialización que después se replican en otros contextos, por ejemplo, el escolar, el social, el laboral y el de pareja.

\section{Acerca de las mediaciones}

La comunicación y socialización se convierten en elementos importantes, al momento de conversar sobre el uso de la tecnología y las nuevas formas de circulación del conocimiento. Serrano (1977) con su teoría de las Mediaciones Sociales; Vygotsky (1934) y la teoría sobre las Zonas de Desarrollo Próximo y Potencial (ZDP), fundamentadas en aportaciones teóricas acerca de las herramientas psicológicas como son, la mediación e internalización); Watzlawick et al. (1985) y su teoría sobre la Pragmática de la Comunicación Humana; Eco (1968/2004, 1976/ 2000) y sus planteamientos sobre Semiótica y Mediaciones Sociales, así como los autores Von Bertalanffy (1968) con la Teoría General de los Sistemas y Morin $(1990,1999)$ con la Teoría sobre la Complejidad y el Pensamiento Complejo, así como sus urgentes reflexiones en torno al pensar global sobre y de la humanidad (2011) y acerca del futuro de la humanidad (2015). 
Serrano (en adelante mms), catedrático de la Universidad Complutense de Madrid (UCM) es uno de los científicos y epistemólogos de la comunicación, cuyos trabajos han tenido mayor influencia en los estudios realizados en este campo desde los años setenta a nuestros días, principalmente en Europa y América Latina. Los campos disciplinares e investigativos diversos, como por ejemplo, la Comunicación, la Medicina, la Sociología, el Trabajo Social, la Psicología, la Fonoaudiología, la Antropología, la Ingeniería de Sistemas, la Pedagogía, la Historia, entre otros, deben generar sinergias interdisciplinarias para conocer, comprender y promocionar usos favorables, incluyentes y positivos para el desarrollo humanístico y socio-familiar del objeto o problemática abordada en este artículo. En un diálogo intercontinental, estas inquietudes se sintonizan con derroteros investigativos como el del Centro Internacional de Estudios Superiores de Comunicación para América Latina (Ciespalc). Una institución fundada en 1959 que a partir de los años setenta jugaría en el continente hermano un papel muy relevante en la producción de estudios y teoría de la comunicación desde una perspectiva crítica, transformadora y latinoamericana.

Uno de los grandes teóricos de la comunicación del último tercio del siglo veinte y principios del veintiuno, Serrano, cuyo trabajo entró en convergencia y enriqueció la Teoría Crítica Latinoamericana a raíz de la publicación en 1977 de "La Mediación Social" $y$, poco después, la edición revisada y aumentada (1981) de "Teoría de la Comunicación I" y "Epistemología y análisis de la referencia” (1982), se constituyen en alternativas teórico-conceptuales para abordar el problema. En particular, su teoría de la Mediación Social (Serrano, 1977) permite explicar el papel que los Medios de Comunicación Social (MCS) juegan como agentes que actúan entre el nivel de los cambios macrosociales y el nivel de los cambios micro psicológicos y conductuales, contribuyendo al ajuste entre ambos. Esta relación contribuye a la producción y reproducción del orden social bajo las nuevas condiciones de dominación del capitalismo postindustrial o monopolista que arranca al final de la década de los años sesenta del siglo anterior. A través de mediaciones cognoscitivas y estructurales, los medios seleccionan el acontecer relevante y los modelos de representa- ción del mundo y de producción de comunicación, conformando y nutriendo la identidad y hábitos que proporcionan referencias comunes a la sociedad, actuando así sobre la conciencia, generando cohesión social y gestionando conflictos inherentes a este tipo de sociedad (Serrano,1985).

La teoría de la mediación social ofrece un nuevo objeto para las ciencias sociales, a saber, el estudio de la producción, transmisión y utilización de la cultura, a partir del análisis de los modelos culturales y de sus funciones. Comprender las formas en que se transmite la información resulta necesario, cuando la cultura se utiliza como un procedimiento de dominación. Así ocurre en los fenómenos de transculturación, es decir, cuando una sociedad destruye las señas de identidad de otra. También sucede en los procesos de control social, cada vez que se propone una visión preestablecida del mundo y de lo que sucede en el mundo, para influir sobre la conciencia y modos de comportamiento de las personas. Con respecto, a los procesos de mediación en relación con los medios de comunicación, reafirma Moragas (1985). En esta área de la comunicación y las mediaciones sociales, hasta hoy, más de treinta años después del florecimiento teórico -al decir de los antiguos griegos-, su perspectiva de los fenómenos comunicativos realimenta y permite comprender los orígenes de la comunicación y la complejidad de sus relaciones con la naturaleza, la sociedad y la vida, estableciendo de este modo la posibilidad de una ciencia de la comunicación autónoma y, al mismo tiempo, productivamente conectada al conjunto de las ciencias biológicas y sociales (inter y transdisciplinariedad). mms proporciona con su trabajo teórico una explicación específicamente comunicativa de los fenómenos, biológicos y culturales, generados por los procesos de comunicación.

Con respecto a las teorías del aprendizaje y su interdependencia con las mediaciones, resultan relevantes y pertinentes, los aportes de Ausubel (citado por Coll, 1994) desde su teoría e investigaciones acerca del aprendizaje significativo; al igual que Vygotsky (citado por Newman, Griffin \& Cole, 1991) con los conceptos de zona de desarrollo próximo y mediación intersubjetiva y semiótica $y$, de Brunner (1994) quien, basado en la teoría de la mediación de Vygotsky, utiliza la metáfora del 
andamio para significar la necesidad transitoria de las ayudas que los docentes, padres de familia y adultos, prestan al niño en su aprendizaje hasta que logren mayores niveles de autonomía (para el caso, en el uso de los aportes tecnológicos de la información y la comunicación).

Desde el campo de la comunicación en contexto, la idea de acción mediada, y desarrollada entre otros por Freire (2001), Barbero (2006), Schmucler (1995), autores que reivindican que el concepto está atravesado por la dimensión comunicacional. Esto significa que no es posible hablar de educación sin que intervenga la comunicación. Entendida esta como producción en común de sentido, definición que se diferencia de aquéllas que sólo proponen la comunicación como transmisión de sentido unidireccional. Al decir Barbero (2002) que los diferentes modelos comunicacionales subyacen a las diversas matrices culturales y pedagógicas, sugiere observar la comunicación desde adentro de los procesos, prácticas y entornos educativos, familiares y socioculturales, y no como solo una estrategia exterior (de ayuda, modernización o adorno) a lo pedagógico.

\section{Lo educativo en las mediaciones}

Los tres componentes de enseñar y aprender son los docentes los alumnos y el conocimiento. Estos interactúan en un determinado contexto y se implican en un proceso complejo, donde, si bien la centralidad la tiene el sujeto que aprende, la ayuda externa -siguiendo a Vygotsky- cobra especial relevancia teniendo en cuenta la especificidad de la comunicación en la modalidad estudiada o en el proceso requerido. El concepto de mediación es clave para interpretar las formas en que los docentes, padres de familia, o ciudadanos, se involucran o intervienen con la finalidad de desplegar estrategias de enseñanza, asesoría u orientación. Estas estrategias tienen como propósito, no sólo de transmitir información, sino lograr que el educando o hijo, se apropie de determinados conocimientos y formas de usar los desarrollos tecnológicos, de uso masivo, con la expectativa de desarrollar, mejores comprensiones y significados, con un contexto específico y de forma proactiva. Así, posibilitarán el desarrollo de, actitudes, comportamientos, hábitos y competencias saludables e integradoras en las interacciones con los demás.

Lo anterior, deberá lograrse sin desplazar la comunicación esencial-personal, ya que, desde un punto de vista humano y epistemológico, sin el reconocimiento del otro como sujeto capaz de lenguaje y acción no es posible una educación autónoma. Esto permitirá un desarrollo amplio desde el punto de vista cognoscitivo (no lo impuesto, lo meramente repetitivo o por imitación) para compartir, en el reconocimiento dialógico, proyectos de vida en común con otros. De esto se entiende entonces que la educación y la comunicación no pueden separarse. Por tanto, el proceso de mediación es un proceso flexible de educación y comunicación, crítico, constante, constructivo y activo, asumiendo entonces, que educar es comunicar, al mismo tiempo que una acción comunicativa conlleva un matiz educativo y humanizador. Se evidencia y confirma, en la cotidianidad, lo que es una tendencia general percibida, acerca del uso y consumo de los medios tecnológicos indagados por parte de la población adolescente, lo cual se contrasta con la realidad al ver en diversos lugares a los jóvenes en contacto y comunicación, "conectados" como se le llama coloquialmente

En la región Caribe colombiana, se han realizado, dos investigaciones al respecto en la ciudad de Barranquilla (Colombia) y en un lapso de casi dos décadas. A saber, "Caracterización de la relación entre las redes sociales virtuales y la formación de la dimensión humana adolescente" realizada por Escorcia (2015) y "Acción mediadora de la familia frente a la recepción de productos televisivos en niños de tercer y cuarto grado escolar con estudiantes de colegios de estrato socioeconómico seis (alto) en la ciudad de Cartagena, Colombia" desarrollada por Silva y Torres (1998). Además de lo anterior, puede observarse que posiblemente los procesos de lectoescritura se vean afectados, tal como lo plantea Silva Pertuz (2003): los aportes tecnológicos son una variada y compleja proyección del ser humano. Procesos tan vitales y decisorios para la evolución del hombre, como la lecto-escritura-, entran en crisis, se redefinen, viven otras formas en su ejercicio, unas positivas y otras en contravía de aquella. 
En esta dinámica local, regional, nacional e internacional, los diferentes desarrollos tecnológicos, entre los que se cuentan las redes sociales on line (virtuales), servicios de la internet y de la televisión. que se están generando en las relaciones interpersonales y en el comportamiento/intereses/ motivaciones humano/s cotidianos, en diversos ámbitos -individual y colectivo. Para comprender lo anterior, serán importantes los soportes teóricos, entre los cuales se encuentra a Capdevilla (2010), quién indica en su estudio que el fenómeno de las redes sociales ha revolucionado el concepto de relación social clásica y la inversión del tiempo libre. En estas se buscan contactos con aquellos con los quienes se ha perdido trato, se mantienen amistades, se pone al día de la vida de los demás, se conoce a gente nueva o incluso se encuentra trabajo.

Por otra parte, Noguera (2010), en su trabajo señaló que España es uno de los países del mundo con mayor consumo de redes sociales y entre estas, Facebook se presenta como la más importante a nivel internacional. $\mathrm{Si}$ a estos fenómenos unimos el evidente panorama de transformación que está sufriendo el periodismo no es de extrañar que el progresivo acercamiento de algunos medios electrónicos españoles (y colombianos). A esta plataforma, pueda valorarse como una de las vías para localizar y desarrollar nuevos productos periodísticos para la red. En dicho estudio se aborda el comportamiento de los cibernautas españoles más significativos a partir de una observación estructurada.

Según Marín Díaz y García Fernández (2003), en su investigación "La familia e internet, ¿un juego tres bandas?, expresaron: Bartolomé (1998) aborda en su estudio que en algunos países como Estados Unidos, el tema ya ha llegado hasta la elaboración de leyes que luego han sido anuladas por el tribunal supremo; los padres se preocupan por los materiales a los cuales sus hijos pueden acceder desde dentro de su casa; los profesores lamentan que sus alumnos "chatean" en horas de clase. Y en el otro extremo, se interrogan acerca de si ¿existe libertad de expresión en internet?

Finalmente, en su artículo titulado "Educación familiar y mediación televisiva”, Cánovas y Sahuquillo (2010) manifestaron la importancia de tratar de abordar "la compleja influencia del visionario televi- sivo en el proceso de socialización y educación de la infancia y la adolescencia, centrando la atención en la necesidad de considerar a la familia como agencia mediadora-educadora de especial relevancia."

Por otro lado, en la investigación de Escorcia et al., manifiestan que se brindará un valioso aporte a la población conformada por niños, niñas y adolescentes, al igual que a los padres de familia, en la orientación que brindan a sus hijos, respecto del adecuado uso de la tecnología, que incluye los medios televisivos, la internet y las leyes sociales.

Sin embargo, las redes sociales despiertan un debate en torno a la privacidad, los riesgos de estas nuevas relaciones, al estar mucho tiempo conectado con varios de amigos, las formas en que afectan o no con la productividad en el trabajo, el desempeño escolar, la calidad de las interacciones familiares y el desempeño de su rol en la familia y en su entorno social.

Actualmente es de interés en el campo de las Ciencias Sociales y Humanas, el estudio de Medios de Comunicación Social (MCS) y la relación de estos con el comportamiento humano, individual y colectivo. Los estudios, las investigaciones, el abordaje y la formación de agentes educativos (en las familias, los contextos escolares, las diversas formas de asociatividad y sistemas sociales), debe ser responsable, comprometido, serio, por parte de quienes deben asumir, usar o interactuar con los desarrollos-aportes tecnológicos de la manera más adecuada y saludable para orientar el manejo de estas relaciones.

Internet se ha promocionado como una fuente de información, de comercio y de entretenimiento. Una tecnología y unos nuevos servicios que están teniendo un impacto radical en la producción (transformando mercados y contextos de distribución) y en particular en el consumo. Este consumo, ha generado una tendencia que ha ido acompañada y en cierto modo ha sido generada, por una retórica mediática para su promoción y popularización. El alto nivel de accesibilidad y la oferta diversa de servicios disponibles en la red, han hecho que se empiece a hablar de un riesgo potencial por sobreuso. Se plantea también la posible presencia de cambios en el estado de ánimo cuando empieza la actividad, así como la existencia de inquietud 
e irritabilidad cuando no se está conectado o se es interrumpido durante la conexión y la aparición de recaídas después de intentos por interrumpir o controlar la frecuencia de acceso a la red.

La relación de los MCs (productos y servicios en la televisión y la internet), particularmente en lo referente a las redes sociales es compleja y polidimensional.

Gadamer (2001) señala que los grandes avances científicos y tecnológicos implican la aparición de otros problemas cuya solución supera el campo de estos saberes. Algunos problemas son nuevos, se han gestado al introducir la aplicación del conocimiento científico y de la tecnología a las condiciones de la existencia cotidiana del ser humano. Pero otros se han exacerbado; es decir, aquellas situaciones que a través de la historia de la humanidad ni la ciencia ni la tecnología han sido capaces de ofrecer una solución satisfactoria; este es el caso de la "tecnologización" o "tecnotronización" de la comunicación y de las interacciones cotidianas en muy variados ámbitos. Lo antes expuesto requiere de perspectivas y enfoques teóricos para aproximarse con fundamento al fenómeno abordado.

\section{De las mediaciones al abordaje familiar}

Hablar de mediación es aludir a uno de los pilares de la enseñanza, en palabras de Edelstein (2002) “... la enseñanza como actividad intencional pone en juego un complejo proceso de mediaciones orientados a imprimir explícita o implícitamente algún tipo de racionalidad a las prácticas que se generan al interior del aula". Así mismo en las relaciones interpersonales, más aún en los tiempos actuales, donde los recursos tecnológicos y el manejo de sus contenidos permean estas interacciones. Para hablar de dicho proceso se parte de la categoría mediación pedagógico comunicacional, haciendo énfasis en la enseñanza, el diálogo y el aprendizaje que se espera, como acto comunicativo o en una comunicación con intencionalidad educativa, en donde tanto los aportes pedagógicos como las cuestiones de índole comunicacional se unen para dar sentido a las prácticas del enseñar y el aprender.

Según Frawley (1999), Moll (1993) y Dixon-Krauss, plantean que la mediación puede ser entendida como el conjunto de instrumentos de carácter cognitivo, físico o instrumental que hacen posible que la actividad cognitiva se desarrolle y logre las metas propuestas. En pocas palabras, la mediación pedagógica en este aspecto propone que los instrumentos externos nos ayudan a que se puedan representar internamente, también este mismo proceso se aplica a los procesos de interacción familiar, por medio de las instrucciones del profesor, de preguntas, observaciones y guiadas por el docente.

De esta manera, la mediación familiar de los padres con sus hijos, entre más temprana sea la edad de estos, mayor se espera sea el efecto de la labor orientadora y educativa. De aquí que el acompañamiento familiar sea vital en el global mundo de hoy, en el caso específico de las mediaciones familiares frente a los productos y servicios televisivos, de internet y las redes sociales on line. El auge de las nuevas tecnologías, y de manera especial la facilidad que tienen los niños y jóvenes, para acceder a ellas, al. Silva y Torres (1998), refiriéndose a los servicios televisivos, y que obviamente por su estrecha relación, con otros medios de comunicación, como el internet y las redes sociales online, se incluyen en esta categoría, "han tenido fuertes implicaciones en la dinámica familiar, hasta el punto que, en ocasiones, disminuye y anula la comunicación, afecta horarios, comidas, lenguaje, frecuencia en la interacción con las amistades, distribución del tiempo y muchas otras formas de relación familiar". A lo anterior se suman los cambios socioculturales y de valores en las nuevas generaciones, varios de ellos ocasionados por los nuevos roles asumidos por los padres y de manera especial por las madres, quienes, para laborar, como una contribución a la economía del hogar, dejan sus hijos e hijas, encomendados generalmente, a personas que en la mayoría de los casos no reúnen los requisitos, para aportarles a su buena crianza.

Con respecto a esto, Silva (2003) planteó algunas características que los orientadores o familiares necesitan para cumplir con las tareas y las necesidades de los miembros de la familia. Esto es profundizar en los valores permanentes que se pueden vivir en la familia; reflexionar sobre los procedimientos y medios para lograrlo; conocer las influencias del contexto social; considerar distintos procedimientos 
para que padres e hijos puedan lograr un desarrollo intra y extrafamiliar autónomo y contextualizado. Así mismo, estudiar cómo la familia puede aumentar y cualificar su influencia favorable en la sociedad y en doble vía. Todo lo anterior, se evidencia en los resultados que emergen en la investigación sobre la relevancia del papel mediador y especialmente interventor, que corresponde a las familias, en el proceso de información, educación y formación de los hijos desde la infancia, durante la adolescencia y hasta llegar a la adultez, en lo pertinente a los productos y servicios televisivos, de internet y las redes sociales online.

Lo anterior permitió plantear el siguiente interrogante: ¿Qué posibilidades formativas ofrece la mediación pedagógica en el sistema familiar frente a los contenidos de información ofertados en internet y posibilitados por las redes sociales on line? Así mismo, los objetivos que orientaron la investigación fueron: i) describir la existencia o no de mediaciones familiares en el consumo de productos $y$ servicios televisivos, de internet y desde las redes sociales; ii) Identificar los productos y servicios más utilizados por los estudiantes universitarios y iii) Determinar la existencia del conocimiento de concepto sobre el proceso de mediación familiar.

\section{Metodología}

Esta fue una investigación de tipo Mixto, donde predominó el énfasis en la descripción de los resultados, con características cuantitativas obtenidas a través del cuestionario y fundamentada en el paradigma interpretativo-comprensivo sobre el cual Heidegger (1975) se refiere como, la interpretación de la interacción social, desde la cual propone deben estudiarse las acciones, circunstancias y significados que las personas otorgan cuando interactúan en distintas situaciones y desde las realidades sociales en las cuales viven. Esto hace referencia a un proceso constante $y$, los significados que se les atribuyen, pueden modificarse por otros u otras interacciones. Dichos cambios son importantes para lo que se denomina el método del interaccionismo simbólico. Blumer (1969) plantea que la producción del conocimiento en el trabajo y la cotidianidad social se basa en gran parte en la interpretación, expresa además que todas sus perspectivas se pueden unir y complementar para así llegar a un conocimiento profundo, contextual y claro sobre el fenómeno, individuo o proceso que está siendo estudiado.

De esta manera, el diseño de investigación descriptivo permitió observar y reflexionar sobre las mediaciones pedagógicas que son posibles en los contextos familiares y educativos frente a la recepción y consumo de productos y servicios ofertados por la televisión, la internet y las redes sociales on line. Los participantes en el estudio fueron estudiantes universitarios entre los 16 y 24 años de edad de diversos programas, escogidos a través de la técnica de muestreo no probabilístico a conveniencia del investigador. Las técnicas de recolección de información utilizada fueron mixtas, siendo estas la observación participante y el cuestionario, para el análisis de la información se utilizó la triangulación de los resultados de la observación participante y de los cuestionarios.

\section{Resultados}

Los resultados obtenidos en cuanto a las características de edad, sexo y procedencia fueron los siguientes. Barranquilla, como distrito capital del Atlántico, arrojó el mayor porcentaje de residencia (83\%) o de procedencia de los estudiantes universitarios que participaron en esta investigación. La mayor parte de los estudiantes universitarios que respondieron, fueron de sexo femenino $85 \%$, masculino $15 \%$, en el rango de edad de $16-18$ años $38 \%$, en el de $19-2136 \%$ y en el rango de $22-24$ años $18 \%$.

Por otro lado, en los resultados asociados con los conceptos y usos de las redes sociales, páginas y otros medios de comunicación masiva, encontramos lo siguiente: para un $54 \%$ de los encuestados, las redes sociales tenían como propósito central la comunicación entre personas y casi por unanimidad (99\%), las personas cuestionadas, opinaron que el Internet es un medio muy utilizado en la casa y en el contexto académico. El $99 \%$ de los encuestados opinó que ha utilizado en la casa y en el contexto de estudio el Internet. La mayoría de los encuestados (96\%), manifiesta que tiene claro el concepto de Tv digital, redes sociales on line e Internet. 


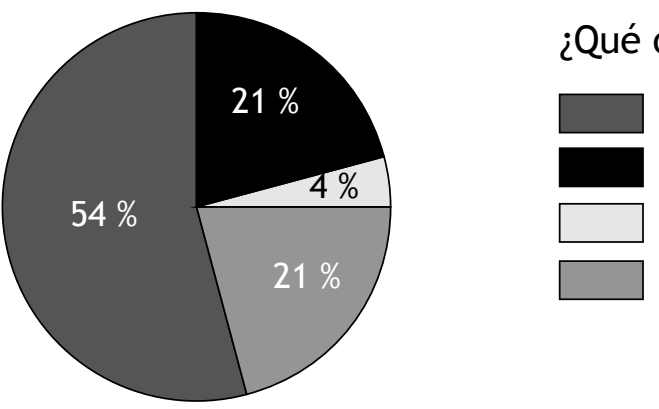

¿Qué opinión tiene de las redes sociales?

Figura 1. Opinión de las redes sociales.

Fuente: elaboración propia.

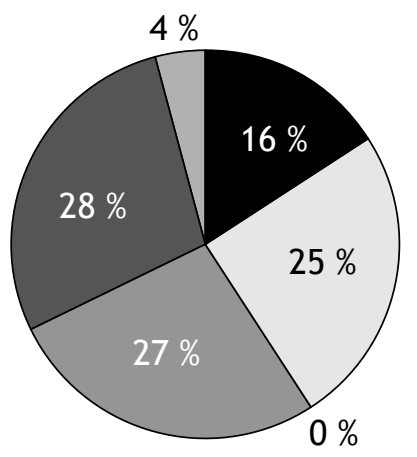

¿Cuál de estos páginas utilizada comúnmente?

a. Son educativas

b. Funcionan para el entretenimiento

c. Tienen como fin la comunicación entre personas

Otra(s) explique su respuesta

Figura 2. Páginas utilizadas comúnmente.

Fuente: elaboración propia.

Estos resultados nos acercaron al objetivo de identificar los productos y servicios más utilizados por los estudiantes universitarios. Sin embargo, pudimos notar que al ser estudiantes universitarios, se esperaría que las páginas más utilizadas fueran académicas de consulta para sus estudios. Por el contrario, es posible que los estudiantes prefieren la información en formatos más visuales y auditivos, como son los videos que comúnmente se encuentran en YouTube.
De la misma forma, nuestros resultados nos permiten pensar en la disposición de la familia para realizar una mediación pedagógica de los contenidos. Sin embargo, los resultados relacionados con el objetivo específico de determinar la existencia del conocimiento de concepto sobre el proceso de mediación, la figura3, nos muestra que para los encuestados, está más relacionado en un $77 \%$ como una intervención para la solución de un conflicto y un $14 \%$ manifiesta que es trasmitir ayuda a una persona necesitada.

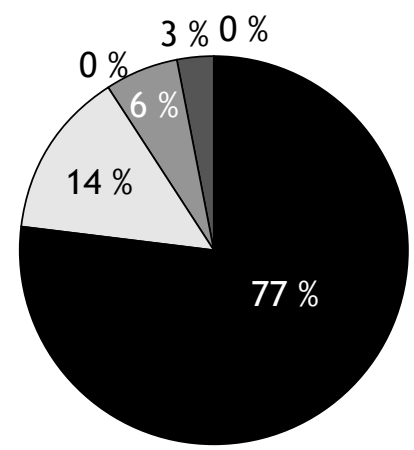

\section{¿Qué concepto tiene usted sobre mediación?}

Figura 3. Concepto acerca de mediación.

Fuente: elaboración propia.

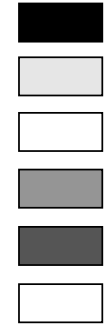

a. Intervención en la solución de un conflicto

b. Transmitir ayuda a una persona necesitada

c. Terminar con un problema

d. Otra(s) explique su respuesta

Sin respuesta

N.N. anter 
Esto se plantea de manera independiente a la tipología familiar con la cual conviven los participantes de la investigación, pero que nos permite, un análisis en relación con las características de la comunicación.

En la figura 4, se observa, los diferentes tipos de familia, prevaleciendo más la nuclear con un $68 \%$.

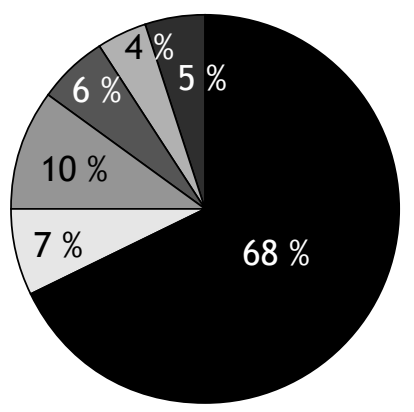

Figura 4. Tipología Familiar.

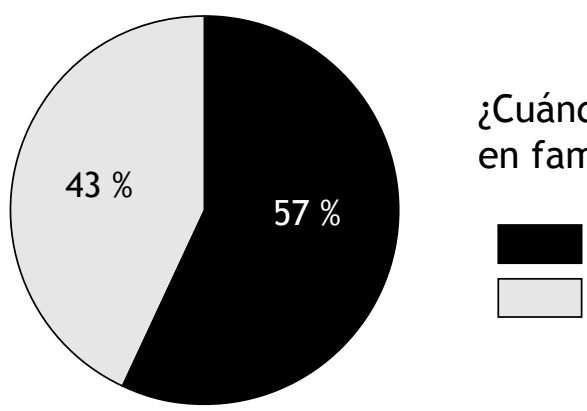

Figura 5. Conversaciones cuando ven tv.

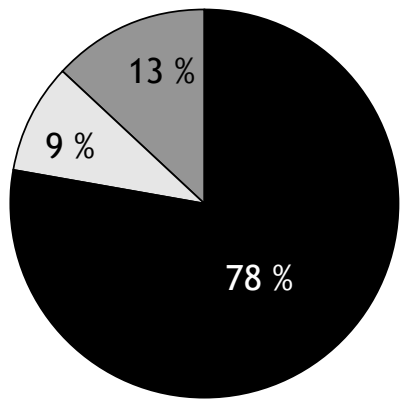

¿Con que frecuencia se reúne la familia para ver televisión con los miembros que la componen?

Figura 6. Sobre la frecuencia con que la familia se reúne para ver tv.
La figura 5 muestra que el $57 \%$ de las personas encuestadas manifiesta que luego de ver TV, conversan con su familia acerca de los contenidos del mismo.

El $78 \%$ de los encuestados opina que algunas veces ven televisión con los miembros de la familia (Figura 6).

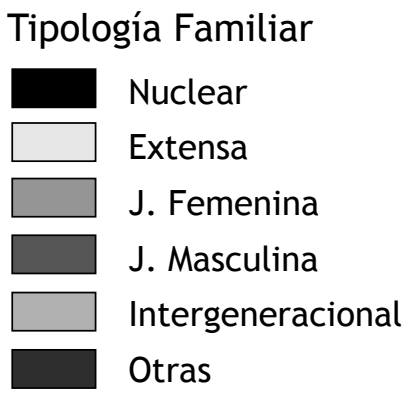

Tipología Familiar

J. Femenina

ntergeneracional 


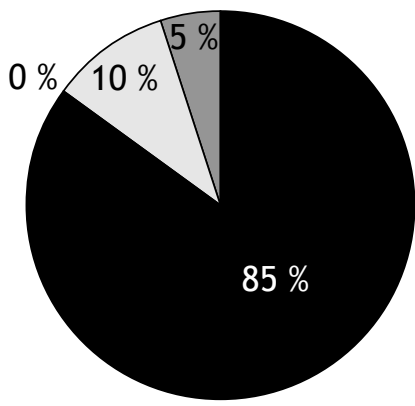

Figura 6. Resolución de conflictos
¿Si tiene un conflicto en su familia y en la universidad, de qué manera interviene en la solución?

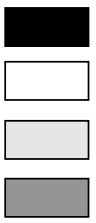

a. Resuelves el conflicto mediante el diálogo

b. Agredes verbal o físicamente a la persona involucrada

c. Buscas ayuda de un mediador

d. Otra(s) explique su respuesta
Cada miembro de la familia consume información y contenidos desde diferentes plataformas y páginas de internet, así que la mediación de estos contenidos en parte se puede ver limitada, siendo la televisión, un espacio de integración y que a la vez se presenta como propicio para iniciar la mediación pedagógica, sea de manera consciente o no.

Igualmente, nos da una pista sobre la calidad de la comunicación y de la estrategia más frecuente que se utiliza en casa y que se replica en contextos sociales como la universidad o con los amigos.

De los encuestados, el $85 \%$, manifiestan que ante los conflictos de tipo familiar o de tipo educativo, se debe recurrir al dialogo. De igual forma está claro para la mayoría que la mediación es una intervención benéfica para la solución de los conflictos. $\mathrm{El}$ $85 \%$ de los encuestados manifiesta que ante un conflicto en la familia y en la universidad, lo resuelven mediante el diálogo.

Podría pensarse, entonces, que los procesos de mediación en los subsistemas familiares, escolar y sociales en general pudiesen estar influenciados por el tiempo que dedican los niños, jóvenes y adultos al consumo de los contenidos de estos mcs en la década actual. Estos parecen ser que han modificado los espacios de interacción familiar, escolar y social en general, propiciando de esta manera, nuevas formas de comunicación y mecanismos de adaptación a estos nuevos estilos de interacción. No se puede desconocer que las tecnologías de información y comunicaciones (TIC) o nuevas tecnologías de información y comunicaciones (NTIC), son un fenómeno influyente, desde la perspectiva de ser herramientas que en múltiples aspectos aportan para el adecuado y saludable desarrollo del proceso cognoscitivo de los niños, jóvenes y adultos, para su crecimiento personal de cara al progreso y aporte a la sociedad.

\section{Conclusión}

Esta forma de asumir los contenidos y nuevas formas de interacción será exitosa o no en la medida que las familias y las escuelas, sepan generar estrategias de mediación funcionales. En la medida que la familia se contextualice y acompañe a sus miembros en el proceso de aprendizaje para el manejo de las tecnologías y su adecuada aplicabilidad para el beneficio de quienes la utilizan. Estas nuevas tecnologías no compiten con los actuales procesos educativos y de formación integral. Poder, identificar las formas de interacción familiar, podría ser la clave para iniciar este proceso. Nos encontramos con familias cuya pauta de interacción se pudo percibir como "tubos de cristal" (se les ve juntos a la vez que muy poco comunicativos, ensimismados en la relación con el dispositivo móvil, con comportamientos de "desprendimiento" o desarraigo familiar. Lo que implicaría una oportunidad para plantear estrategias de comunicación familiar, que permitan una injerencia más directa de los padres o cuidadores en estos contenidos, teniendo en cuenta el ciclo vital individual y familiar. 


\section{Referencias}

Escorcia J. L. (2015). Caracterización de la relación entre las redes sociales virtuales y la formación de la dimensión humana adolescente. (Trabajo de Maestría en Educación). Universidad Simón Bolívar, Barranquilla, Colombia.

Silva, M. \& Torres, M. (1998). Acción mediadora de la familia frente a la recepción de productos televisivos en niños de tercer y cuarto grado escolar. (Trabajo de Maestría en Desarrollo Familiar). Universidad del Norte Barranquilla, Colombia.

Silva, M. \& Torres, M. (2006). Acción Mediadora de la familia frente a la recepción de productos televisivos en niños de tercer y cuarto grado escolar. Revista Psicogente,

Edelstein, G. (2002). Problematizar las prácticas de la enseñanza. Perspectiva. 20(2), 21-30

Capdevilla, D. (2010). Las Redes Sociales. Tipología, uso y consumo de las redes 2.0 en la sociedad digital actual. Madrid: Facultad de Ciencias de la Información de la Universidad Complutense de Madrid.

Noguera, J. M. (2010). Redes sociales como paradigma periodístico. Medios españoles en Facebook, Revista Latina de Comunicación Social, 65.

Marín, Díaz. V. \& García Fernández, M. D. (2003). La familia e internet, ¿un juego tres bandas? Córdoba: Comunicar 21: Tecnologías en la era de la comunicación.

Bartolomé, A. (1998). Familia y escuela: El miedo a internet. Barcelona: Comunicar 10.

Cánovas, P. \& Sahuquillo, P. (2010). Educación familiar y mediación televisiva. Universidad de Valencia. Valencia

Escorcia L., García A., \& Silva M. (2015). Mediaciones familiares, escolares y socio-culturales frente a los productos y servicios televisivos de internet y las redes sociales online, investigación en curso.
Silva, M. (2003). Desde los tres robles. Cartagena: Editorial Ideas gráficas.

Gadamer, H. (2001). Fenomenología, hermenéutica y metafísica, en: El giro hermenéutico, Madrid: Cátedra 1998, p. 29

Serrano, M. M. (1977). La mediación social. Madrid: Akal

Vygotsky, L. (1934). Pensamiento y lenguaje.

Watzlawick, P. y Cols. (1985). Teoría de la Comunicación Humana. Barcelona: Editorial Herder.

Eco, U. (2004). Apocalípticos e integrados. México: Lumen / Tusquets. (Trabajo original publicado en 1968)

Eco, U. (2000). Tratado de semiótica general. España: Lumen. (Trabajo original publicado en 1976)

Von Bertalanffy, L. (1968). Teoría General de Sistemas. Fundamentos, desarrollo y aplicaciones. Nueva York.

Von Bertalanffy, L. (1975). Perspectiva de la teoría general de Sistemas. Estudios científicos y filosóficos. E. Taschdjian (eds.), New York, E-U

Morin, E. (1990). Introducción al pensamiento complejo.

Morin, E. (1999). La inteligencia de la complejidad.

Morin, E. (2011). La Vía. Para el futuro de la humanidad.

Morin, E. (2015). Pensar global. La humanidad y el universo.

Moragas, D. M. (1985). La mediación social y los enfoques de la teoría de la comunicación. Barcelona.

Freire P. (1984). La importancia de leer y el proceso de liberación. México: Siglo XXI Editores.

Freire P. (1996). Pedagogía de la autonomía. México: Siglo XXI editores. 
Barbero J. (2006). La educación desde la comunicación. Bogotá: Editorial Norma.

Schmucler, H. (1995). El imperio de la Información como imperio de la Banalidad. En: Comunicación y Educación como campos problemáticos desde una perspectiva epistemológica. Paraná: Facultad de Ciencias de la Educación, Universidad Nacional Entre Ríos (UNER).

Diccionario de la Lengua Española. (2015).

Husserl, E. http://www.philosophica.info/voces/ husserl/Husserl.html

Heidegger, M. (1975). La pregunta por la cosa. Buenos Aires: Editorial Alfa Argentina. (Traducido por: Eduardo García Belsunce \& Zoltan Szankay).

Blumer, H. (1982). El Interaccionismo simbólico, perspectiva y método. Barcelona: Hora D.L.

Wolf, M. (1987). La investigación de la comunicación de masas, Barcelona: Paidós.
Deslauriers, J.P. (2004). La investigación cualitativa. Guía Práctica. Universidad Tecnológica de Pereira - Rudecolombia.

Ferraroti, F. (1981). Sobre la autonomía del método biográfico, D. Bertaux (Ed). Biografía y Sociedad: La Historia de Vida. Enfoque en las Ciencias Sociales, Beverly Hills, California.

Ferraroti, F. (1983). Historia de las historias de vida. El método biográfico en las ciencias Sociales. Meridien. París.

Pick, S. (1992). La recolección de la información.

Sandín, M. P. (2003). "Investigación Cualitativa en Educación. Fundamentos y Tradiciones". Mc Graw and Hill Interamericana de España, Madrid .

Salanova, E. (2013). Implicaciones creativas de la educación intercultural. El encuentro de las culturas a través de los medios de comunicación -capítulo- (Libro "Interculturalidad y Neocomunicación”). Encarnación Soriano Ayala (coordinadora). La Muralla. Madrid. 